\title{
ASYMPTOTIC INTERNAL WORKING SURFACES OF PERIODICALLY VARIABLE JETS
}

\author{
A. C. Raga ${ }^{1,2}$, J. Cantó ${ }^{3}$, and A. Castellanos-Ramírez ${ }^{3}$ \\ Received July 11 2020; accepted November 62020
}

\begin{abstract}
We present a derivation based on the "center of mass formalism" of the asymptotic behaviour of internal working surfaces produced in a variable HerbigHaro $(\mathrm{HH})$ jet. We obtain the general solution for an arbitrary periodic ejection time-variability, and then show examples for a limited set of functional forms for the velocity and density time-evolutions. Finally, we derive a prescription for obtaining the time-averaged mass loss rate from observations of knots along an $\mathrm{HH}$ jet (based on the asymptotic solution), and apply it to derive the mass loss rate of the $\mathrm{HH} 1$ jet.
\end{abstract}

\section{RESUMEN}

Presentamos una derivación basada en el "formalismo de centro de masa" del comportamiento asintótico de superficies de trabajo internas producidas en un yet Herbig-Haro $(\mathrm{HH})$ variable. Obtenemos la solución general para una eyección periódica arbitraria, y después mostramos ejemplos para un conjunto limitado de formas funcionales para la evolución temporal de la velocidad y la densidad. Finalmente, derivamos una prescripción para calcular la tasa de pérdida de masa promedio de observaciones de los nudos a lo largo de un yet HH (basada en la solución asintótica), y la aplicamos para derivar la pérdida de masa del yet de HH 1.

Key Words: Herbig-Haro objects — ISM: individual objects: HH 1 - ISM: jets and outflows - ISM: kinematics and dynamics — stars: formation stars: winds, outflows

\section{INTRODUCTION}

The suggestion that the knotty structures in astrophysical jets could be the result of a time-dependent ejection was first made in the context of extragalactic jets (see, e.g., Rees 1978; Wilson 1984; Roberts 1986). However, the theory of variable jets has been mostly developed and applied in the context of Herbig-Haro (HH) jets from young stars.

Raga et al. (1990) apparently first pointed out in an explicit way that the structures observed in $\mathrm{HH}$ jets could be easily modeled as "internal working surfaces" produced by an ejection velocity variability with a hypersonic amplitude (though the general idea that $\mathrm{HH}$ knots are the result of a variability of the ejection hovers around in the literature of the late 1980 's). Since then, a relatively large number of pa-

\footnotetext{
${ }^{1}$ Instituto de Ciencias Nucleares, UNAM, México.

${ }^{2}$ Inst. de Investigación en Ciencias Físicas y Matemáticas, USAC, Guatemala.

${ }^{3}$ Instituto de Astronomía, UNAM, México.
}

pers has been written on numerical simulations and analytic models of variable ejection $\mathrm{HH}$ jets, as well as comparisons with observations (three relatively recent examples are Teşileanu et al. 2014; Hansen et al. 2017; Castellanos-Ramírez et al. 2018).

Kofman \& Raga (1992) and Raga \& Kofman (1992) studied analytically the asymptotic regime reached by internal working surfaces at large distances from the outflow source. They noted that the internal working surface shocks (see Figure 1) asymptotically have shock velocities that scale as $1 / x$ and pre-shock densities with the same dependence on distance $x$ from the source. Approximating the emission from these shocks with the predictions from plane-parallel shocks, Raga \& Kofman (1992) showed that the asymptotic working surface model predicts a [S II] line intensity vs. $x$ decay that agrees surprisingly well with observations of the HH 34 jet. More recently, Raga et al. (2017) showed that the 
successive knots along the HH 1 jet have the predicted [S II] intensity vs. position dependence, and also that individual knots follow the predicted behaviour as a function of time, following the increase in $x$ that results from their motion away from the outflow source.

Kofman \& Raga (1992) and Raga \& Kofman (1992) found the asymptotic regime by considering a "ram-pressure balance" equation of motion for the internal working surfaces. This equation of motion is valid for the case in which the gas that goes through the working surface shocks is ejected laterally in an efficient way, and does not remain within the working surface. Though these authors determined the form of the position dependence of the shock velocities and pre-shock densities of the internal working surfaces, they were unable to relate the proportionality constants of these dependencies to the functional form of the ejection velocity and density.

In this paper, we study the asymptotic regime (of internal working surfaces at large distances from the outflow source) using the "center of mass" equation of motion of Cantó et al. (2000). This equation of motion is valid for internal working surfaces in which a large part of the gas passing through the shocks stays within the working surface. The theoretical attraction of this formalism is that it generally leads to full (though possibly quite complex) analytic solutions (see, e.g., Cantó \& Raga 2003).

The paper is organized as follows. In $\S 2$ we provide a summary of the "center of mass formalism" of Cantó et al. (2000), giving the equation of motion for the internal working surfaces and the free-flow (velocity and density) solution for the continuous jet beam segments between the working surfaces. In $\S 3$, we derive the full asymptotic solution for large distances from the outflow source. In $\S 4$, we derive the properties of the working surfaces for a limited set of chosen ejection velocity and density variabilities. In $\S 5$, we calculate the $\mathrm{H} \alpha$ and red [S II] positiondependent luminosities of the asymptotic working surfaces. In $\S 6$, we discuss the "inverse problem" of taking the observed properties of a knot (in particular, the spatial velocity and line luminosity of a given knot, and the knot position and knot spacing) and deducing the mean mass loss rate of the outflow. In $\S 7$, we use this inverse problem to deduce the mass loss rate of the HH 1 jet. Finally, the results are summarized in $\S 8$.

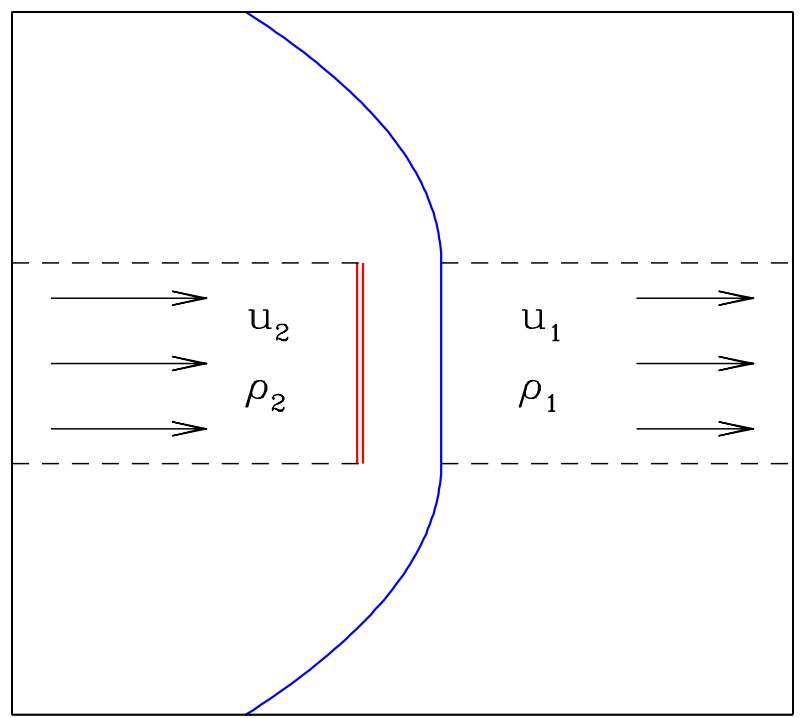

Fig. 1. Schematic diagram of an internal working surface produced by the interaction of slower material (of velocity $u_{1}$ and density $\rho_{1}$ ) with faster material (of velocity $u_{2}$ and density $\rho_{2}$ ) ejected at later times. The working surface has two shocks. the bow shock (blue, solid line) and the "jet shock" (double, solid red line). The dashed lines represent the outer boundary of the jet beam. The color figure can be viewed online.

\section{EQUATION OF MOTION FOR AN INTERNAL WORKING SURFACE}

This section is a short summary of the "center of mass equation of motion" for working surfaces derived by Cantó et al. (2000). The idea embodied by this formalism is as follows:

- in a hypersonic jet (or wind), in the absence of shocks the fluid parcels are free-streaming, preserving their initial ejection velocity $u_{0}$,

- when shocks form due to "catching up" of faster parcels ejected at later times with slower parcels ejected at earlier times, "internal working surfaces" are formed (see Figure 1). These working surfaces are assumed to be compact (with extents along the outflow direction which can be neglected), so that each of them has a single, time-dependent distance from the source $x_{w s}$,

- if one assumes that all of the mass entering through the two working surface shocks stays in a region close to the working surface (an assumption that is correct for a spherical wind, and might also be appropriate for radiative jets), then: 
- with this "mass conservation" condition, a working surface can be seen as a particle formed by the coalescence of fluid parcels, with the mass and momentum of the coalesced parcels. Then, the position $x_{w s}$ of the working surface will be equal to the position $x_{c m}$ of the center of mass of the fluid parcels if they had continued freestreaming without coalescing.

Cantó et al. (2000) showed that this center of mass can be calculated as a function of the ejection velocity and density history in a direct way, leading to analytic solutions for the time-dependent positions and velocities of the successive internal working surfaces. Here, we summarize their results.

Let us assume an arbitrary, periodic variation $u_{0}(\tau), \rho_{0}(\tau)$ of the ejection velocity and density. This periodic ejection variability produces a chain of internal working surfaces, and we consider the timedependent position

$$
x_{c m}(t)=\frac{\int_{\tau_{1}}^{\tau_{2}} x(t, \tau) \rho_{0}(\tau) u_{0}(\tau) d \tau}{\int_{\tau_{1}}^{\tau_{2}} \rho_{0}(\tau) u_{0}(\tau) d \tau},
$$

of the centre of mass of the material within one of the working surfaces. In this equation, $t$ is the present time, and $\tau \leq t$ is the "ejection time" at which the fluid parcels were ejected. The position $x(t, \tau)$ of the free-streaming fluid parcels is given by the freestreaming flow condition

$$
x(t, \tau)=(t-\tau) u_{0}(\tau) .
$$

The $\tau_{1}$ and $\tau_{2}$ values in equation (1) are the ejection times of the fluid parcels which are now entering the working surface from the downstream and upstream directions (respectively), and correspond to two successive roots of the equation:

$$
x_{c m}=\left(t-\tau_{1,2}\right) u_{0}\left(\tau_{1,2}\right) \text {. }
$$

We also note that the density of a free-streaming jet with a position-dependent cross section $\sigma(x)$ is given by:

$$
\sigma(x) \rho(x, t)=\frac{\sigma_{0} \rho_{0}(\tau) u_{0}(\tau)}{u_{0}(\tau)-(t-\tau) \dot{u}_{0}(\tau)},
$$

where $\sigma_{0}$ and $\rho_{0}(\tau)$ are the ejection cross section and density, respectively, and $\dot{u}_{0}(\tau)=d u_{0} / d \tau$. This solution for the density can be straightforwardly obtained by inserting the free flow condition (2) into the appropriate continuity equation.

\section{THE ASYMPTOTIC REGIME}

For large distances from the source, most of the ejected material has already entered the working surfaces, so that the ejection time-interval of the material entering the working surface from the upstream and downstream directions becomes $\tau_{2}-\tau_{1} \approx \tau_{p}$, where $\tau_{p}$ is the period of the ejection variability. In this regime, the $\tau_{1} \rightarrow \tau_{2}$ interval of the integrals can therefore be replaced by the $-\tau_{p} / 2 \rightarrow \tau_{p} / 2$ interval. Equation (1) then becomes:

$$
x_{c m}=\left(t-\tau_{a}\right) v_{a},
$$

where

$$
v_{a}=\frac{\int_{-\tau_{p} / 2}^{\tau_{p} / 2} \rho_{0}(\tau) u_{0}^{2}(\tau) d \tau}{\int_{-\tau_{p} / 2}^{\tau_{p} / 2} \rho_{0}(\tau) u_{0}(\tau) d \tau},
$$

is the (constant) asymptotic velocity of the working surface and

$$
\tau_{a}=\frac{\int_{-\tau_{p} / 2}^{\tau_{p} / 2} \tau \rho_{0}(\tau) u_{0}^{2}(\tau) d \tau}{v_{a} \int_{-\tau_{p} / 2}^{\tau_{p} / 2} \rho_{0}(\tau) u_{0}(\tau) d \tau},
$$

is an average ejection time of the material that lies within a given internal working surface. Clearly, by choosing to carry out the integrals over the $-\tau_{p} / 2 \rightarrow \tau_{p} / 2$ range we are choosing the internal working surface formed by the material ejected in this ejection time interval.

Therefore, regardless of the form of the periodic ejection velocity and density variability, at large distances from the source the working surfaces travel at a constant velocity, which is given by equation (6). It is also possible to obtain the shock velocities of the working surface shocks in the following way.

At large distances from the source, the material in the continuous segments of the jet corresponds to a small range of ejection times around $\tau_{n}$, where the index $n$ numbers the successive continuous segments. The ejection time $\tau_{n}$ is determined by the condition

$$
u_{0}\left(\tau_{n}\right)=v_{a},
$$

where one has to choose the root with $\dot{u}_{0}\left(\tau_{n}\right)<0$, and $v_{a}$ is given by equation (6). Clearly,

$$
\tau_{n+1}=\tau_{n}+\tau_{p},
$$

and the free-streaming flows on the two sides of the working surface have linear velocity vs. position relationships, giving velocities

$$
u_{1}=\frac{x_{c m}}{t-\tau_{n}}, \quad u_{2}=\frac{x_{c m}}{t-\tau_{n+1}},
$$


immediately down- and up-stream of the working surface.

Using equation (9), we have

$$
t-\tau_{n+1}=\left(t-\tau_{n}\right)(1-\epsilon), \text { with } \epsilon=\frac{\tau_{p}}{t-\tau_{n}},
$$

with $\epsilon \ll 1$ in the asymptotic regime.

We can then use equations (5), (10) and (11) to calculate the velocity jump accross the working surface:

$$
\Delta u=u_{2}-u_{1}=\frac{v_{a}^{2} \tau_{p}}{x_{c m}},
$$

where we have carried out a first order expansion in $\epsilon$ (see equation 11).

Also, the free-streaming flow density integral (4), when evaluated in $\tau_{n}$ gives:

$$
\rho_{1,2} \approx \frac{\rho_{0}\left(\tau_{n}\right) \sigma_{0}}{\sigma\left(x_{c m}\right)\left[1-\left(t-\tau_{n}\right) \frac{d \ln u_{0}}{d \tau}\left(\tau_{n}\right)\right]},
$$

where we can calculate both upstream and downstream densities using $\tau_{n}$, given that in the asymptotic regime we have $\epsilon \ll 1$ (see equation 11). In this equation, $\sigma_{0}$ is the ejection cross section and $\sigma\left(x_{c m}\right)$ the cross section at the position of the working surface. Equation (13) can be further simplified by noting that

$$
-\left(t-\tau_{n}\right) \frac{d \ln u_{0}}{d \tau}\left(\tau_{n}\right) \approx \frac{t-\tau_{n}}{\tau_{p}}=\epsilon^{-1},
$$

and therefore, in the asymptotic, $\epsilon \ll 1$ regime the first term in the denominator of equation (13) can be neglected. In this way, we obtain

$$
\rho_{1,2} \approx-\frac{\rho_{0}\left(\tau_{n}\right) \sigma_{0} u_{0}\left(\tau_{n}\right)}{\sigma\left(x_{c m}\right) \dot{u}_{0}\left(\tau_{n}\right)\left(t-\tau_{n}\right)},
$$

with equal densities on both sides of the internal working surface. The fact that the densities on both sides of the working surface asymptotically approach each other, and that the velocity of the working surface becomes constant, implies that the shock velocities of the two working surface shocks also have the same value. Therefore, the velocity jump $\Delta u$ across the working surface (see equation 12) is divided into two shocks of velocities $\Delta u / 2$. In this way, we see that as the working surface travels away from the outflow source at the asymptotic velocity $v_{a}$, the shocks have velocities that decrease as $1 / x_{c m}$ (see equation 12).

Combining equations (5), (15) and (8) we obtain:

$$
\rho_{1,2}=\frac{\Sigma}{x_{c m} \sigma\left(x_{c m}\right)}
$$

where

$$
\Sigma \equiv-\rho_{0}\left(\tau_{n}\right) \sigma_{0} \frac{v_{a}^{2}}{\dot{u}_{0}\left(\tau_{n}\right)},
$$

is a (positive) constant, $\sigma\left(x_{\mathrm{cm}}\right)$ is the cross section of the jet (at the position of the working surface) and $\rho_{0}$ and $\dot{u}_{0}$ are calculated at the time $\tau_{n}$ at which the material of the asymptotic segments of continuous jet beam were ejected, which is given by equation (8).

\section{EXAMPLES FOR A SINUSOIDAL $U_{0}(\tau)$ AND TWO SIMPLE FORMS OF $\rho_{0}(\tau)$}

\subsection{Ejection Velocity Variability}

For the ejection velocity, we choose a sinusoidal variability:

$$
u_{0}(\tau)=v_{0}+\Delta v_{0} \sin \omega \tau,
$$

with mean velocity $v_{0}$, half-amplitude $\Delta v_{0}$, frequency $\omega$ and period $\tau_{p}=2 \pi / \omega$. The half amplitude $\Delta v_{0}$ lies in the $0 \rightarrow v_{0}$ interval.

\subsection{Constant $\dot{M}$}

We first choose a density variability such that the jet has a time-independent $\dot{M}$. The ejection density then is:

$$
\rho_{0}(\tau)=\frac{\dot{M}}{\sigma_{0} u_{0}(\tau)}=\frac{\dot{M}}{\sigma_{0}\left(v_{0}+\Delta v_{0} \sin \omega \tau\right)},
$$

where $\sigma_{0}$ is the ejection cross section, and where we have used equation (18) for the second equality.

With the chosen $u_{0}(\tau)$ and $\rho_{0}(\tau)$ (equations 18 and 19 , respectively), from equation (6) we obtain

$$
v_{a}=v_{0},
$$

from equation (8) we obtain

$$
\tau_{n}=\tau_{p} / 2+n \tau_{p},
$$

and from equation (17) we obtain

$$
\Sigma=\frac{\dot{M} v_{0} \tau_{p}}{2 \pi \Delta v_{0}} .
$$

In this way, we can calculate the shock velocities $\Delta u / 2$ (see equation 12) and pre-shock densities $\rho_{1}=\rho_{2}$ (see equation 16) of the asymptotic working surfaces as a function of their position $x_{c m}$, the jet cross-section $\sigma\left(x_{c m}\right)$, the (time-independent) mass loss rate $\dot{M}$, and the period $\tau_{p}$, mean velocity $v_{0}$ and half-amplitude $\Delta v_{0}$ of the ejection velocity variability. 


\subsection{Constant $\rho_{0}$}

We now consider the case of a time-independent ejection density $\rho_{0}$. Then, the time-averaged mass loss rate of the ejected jet is $\dot{M}=\sigma_{0} \rho_{0} v_{0}$, where $\sigma_{0}$ is the ejection cross section and $v_{0}$ is the mean velocity of the jet (see equation 18).

Using equation (18) and setting a timeindependent $\rho_{0}$, from equation (6) we obtain

$$
v_{a}=v_{0}\left[1+\frac{1}{2}\left(\frac{\Delta v_{0}}{v_{0}}\right)^{2}\right],
$$

from equation (8) we obtain

$$
\tau_{n}=\frac{\tau_{p}}{2}-\frac{\tau_{p}}{2 \pi} \sin ^{-1}\left(\frac{\Delta v_{0}}{2 v_{0}}\right)+n \tau_{p},
$$

and from equation (17) we obtain

$$
\Sigma=\frac{\dot{M} v_{0} \tau_{p}}{2 \pi \Delta v_{0}} g\left(\frac{\Delta v_{0}}{v_{0}}\right),
$$

with

$$
g\left(\frac{\Delta v_{0}}{v_{0}}\right)=\frac{\left[1+\frac{1}{2}\left(\frac{\Delta v_{0}}{v_{0}}\right)^{2}\right]^{2}}{\sqrt{1-\frac{1}{4}\left(\frac{\Delta v_{0}}{v_{0}}\right)^{2}}} .
$$

If we consider the $\Delta v_{0} / v_{0} \rightarrow 0$ lower limit of the velocity amplitude, we regain the results obtained for the constant mass loss rate case (see $\S 4.2$ ). If we consider the $\Delta v_{0} / v_{0} \rightarrow 1$ upper limit, we obtain:

$$
\begin{gathered}
v_{a}=\frac{3 v_{0}}{2}, \\
\tau_{n}=\frac{5 \tau_{p}}{12}+n \tau_{p},
\end{gathered}
$$

and

$$
\Sigma=\frac{3 \sqrt{3} \dot{M} v_{0} \tau_{p}}{4 \pi \Delta v_{0}} .
$$

Therefore, in the $\Delta v_{0} / v_{0} \rightarrow 1$ large amplitude limit the constant $\rho_{0}$ case gives an asymptotic velocity $v_{a}$ for the working surfaces which is a factor $3 / 2$ larger than the one of the constant mass loss case, and a "density constant" $\Sigma$ larger by a factor $3 \sqrt{3} / 2$.

\section{THE EMISSION OF ASYMPTOTIC WORKING SURFACES}

We now estimate the $\mathrm{H} \alpha$ and red [S II] luminosities of the asymptotic working surfaces as:

$$
L_{\text {line }}=8 \pi \sigma I_{\text {line }}\left(n_{\text {pre }}, v_{s}\right),
$$

where $\sigma$ is the cross section of the jet at the position of the working surface, $n_{\text {pre }}=\rho_{1,2} /\left(1.3 m_{H}\right)$ (where $\rho_{1,2}$ is the pre-working surface shock density, see equation 16$), v_{s}=\Delta u / 2$ is the shock velocity (see equation 12), and $I_{\text {line }}$ is the line flux emerging from one of the two shocks (the factor $8 \pi$ accounting for the fact that we have 2 shocks radiating into $4 \pi$ sterad).

As described in Appendix A, we use the planeparallel, steady shock models of Hartigan et al. (1987) to determine the functional form:

$$
I_{\text {line }}=n_{\text {pre }} f_{\text {line }}\left(v_{s}\right),
$$

with $f_{\text {line }}=f_{H \alpha}$ or $f_{[S I I]}$ determined from fits to the predictions of the plane-parallel shock models (see equations A38 and A39 of Appendix A).

Combining equations (30), (31), (16) and (25), we obtain:

$$
L_{\text {line }}=\frac{4 \dot{M} v_{0} \tau_{p}}{1.3 m_{H} \Delta v_{0}} g\left(\frac{\Delta v_{0}}{v_{0}}\right) \frac{f_{\text {line }}\left(v_{s}\right)}{x_{c m}},
$$

where $\dot{M}$ is the time-averaged mass loss rate (see equation 25) and $v_{s}=\Delta u / 2$ is given by equation (12). Equation (32) is equivalent to equation (34) of Raga \& Kofman (1992), but includes a more general form for the shock velocity dependence of the emission and a full determination of the constants.

For a sinusoidal ejection velocity variability and a density variability such that the mass loss rate is time-independent (see § 4.2), the position-dependent luminosity of the working surface in the $\mathrm{H} \alpha$ and [S II] lines can be obtained by setting $f=f_{H \alpha}$ or $f=f_{S I I}$ (see equations A38 and A39 in Appendix A, respectively) and $g\left(\Delta v_{0} / v_{0}\right)=1$ (see equation 22 ).

For the case of a constant density ejection, the $\mathrm{H} \alpha$ and $\left[\begin{array}{ll}\mathrm{S} & \mathrm{II}\end{array}\right]$ luminosities can be obtained using the $g\left(\Delta v_{0} / v_{0}\right)$ function of equation (26). For $\Delta v_{0} / v_{0} \ll 1$, this function has a value $g\left(\Delta v_{0} / v_{0}\right) \approx 1$.

\section{THE INVERSE PROBLEM}

Several HH outflow systems show chains of quasiperiodic, aligned knots within $\approx 10^{17} \mathrm{~cm}\left(\approx 10^{4} \mathrm{AU}\right)$ of the outflow source. These knots generally have spatial velocities in excess of $\approx 150 \mathrm{~km} \mathrm{~s}^{-1}$ (determined from radial velocity and proper motion studies), and have very low excitation emission line spectrum, with high red [S II] $/ \mathrm{H} \alpha$ and [O I] $6300 / \mathrm{H} \alpha$ line ratios. These line ratios imply relatively slow shock velocities (of $\approx 20-30 \mathrm{~km} \mathrm{~s}^{-1}$ ). 
In the case of the $\mathrm{HH} 1$ jet, this very low excitation is present in all of the observed knots along the HH 1 jet, including the knots that lie closer to the outflow source (observed in the IR, see, e.g., Table 2 of Nisini et al. 2005). The knots formed by a velocity variability with a half-amplitude $\Delta v_{0}$ produce internal working surfaces that rapidly reach peak shock velocities $v_{s} \approx \Delta v_{0}$ (before reaching the asymptotic regime described in $\S 3$ ), as shown, e.g., by Raga \& Cantó (1998) and Cantó et al. (2000). Therefore, the low excitation of all knots along the HH 1 jet (and in particular, the ones closer to the outflow source) indicates that the ejection time variability in HH 34 has a small $\Delta v_{0} / v_{0}$ (where $v_{0}$ is the mean ejection velocity, and $\Delta v_{0}$ is the half-amplitude of the variability, see, e.g., equation 18). A similar situation is found for the $\mathrm{HH} 1$ jet, and for other jets in which all of the knots along the chains close to the outflow source have a very low excitation spectrum (e.g., HH 34, see Podio et al. 2006.

In this section we show how observational determinations of the knot spacing $\Delta x$, and the luminosity $L_{\text {line }}$ of a given emission line and spatial velocity $v_{a}$ of a knot at position $x_{w s}$ can be used to constrain the average mass loss rate of the ejection. We will identify the observed position $x_{w s}$ of the knot with the $x_{c m}$ center of mass position that comes out of our model, so that in the following we will set $x_{c m}=x_{w s}$.

For a low-amplitude sinusoidal ejection velocity variability, both the constant mass loss rate and constant ejection density cases (see $\S 4.2$ and $\S 4.3$ ) give:

$$
v_{a} \approx v_{0} ; \rho_{\text {pre }} \approx \frac{\dot{M} v_{0} \tau_{p}}{2 \pi \Delta v_{0} x_{w s} \sigma\left(x_{w s}\right)},
$$

where $v_{a}$ is the asymptotic working surface velocity, and $x_{w s}$ is the position of a given working surface. The line emission of the working surface is then given by equation (32) with $g\left(\Delta v_{0} / v_{0}\right)=1$.

For a periodic ejection velocity, all of the working surfaces in the asymptotic regime move with the constant velocity $v_{a}$. Therefore, if we observe the spatial velocity $v_{a}$ (determined from proper motion and radial velocity measurements) and knot spacing $\Delta x$, we can obtain the variability period as

$$
\tau_{p}=\frac{\Delta x}{v_{a}} .
$$

We now observe the flux of a given emission line, and using the distance to the object and the extinction (which we assume has also been determined) we can calculate the luminosity $L_{\text {line }}$ of the line. If the observed knot lies at a distance $x_{w s}$ from the outflow source, we first use equation (12) to calculate the shock velocity of the two working surface shocks:

$$
v_{s}=\frac{\Delta u}{2}=\frac{v_{a}^{2} \tau_{p}}{2 x_{w s}}=\frac{v_{a} \Delta x}{2 x_{w s}} .
$$

With our empirical determinations of $L_{\text {line }}, \tau_{p}$ and $v_{s}$, we then invert equation (32) (setting $g=1$, see above) to calculate the average mass loss rate

$$
\dot{M}=\frac{1.3 m_{H} L_{\text {line }} \Delta v_{0} x_{w s}}{4 v_{0} \tau_{p} f_{\text {line }}\left(v_{s}\right)},
$$

where in Appendix A we give analytic forms for the $f_{\text {line }}\left(v_{s}\right)$ functions for the $\mathrm{H} \alpha$ and red [S II] emission. Clearly, in order to calculate the mass loss rate, we need to know the value of the half-amplitude $\Delta v_{0}$ of the ejection velocity variability. If we cannot determine this parameter from other observations, we can set $\Delta v_{0} \approx v_{s}$.

\section{AN APPLICATION TO THE HH 1 JET}

As an example we consider the "HH 1 jet", which points from near the source of the $\mathrm{HH} 1 / 2$ outflow system towards HH 1. Raga et al. (2017) and Castellanos-Ramírez et al. (2018) argue that the intensity vs. position dependence of the knots at distances $>5^{\prime \prime}$ from the source can be modelled as coming from working surfaces in the "asymptotic regime".

We calculate the mass loss rate of the $\mathrm{HH} 1$ jet using the calibrated line fluxes of knot $\mathrm{G}$ by Nisini et al. (2005). At the time of their observations, the $\mathrm{G}$ knot was at $x_{G}=6.5^{\prime \prime}=3.9 \times 10^{16} \mathrm{~cm}$ from the outflow source. From the HST images shown in Raga et al. (2017), we see that the separation between successive knots is $\Delta x_{G} \approx 2^{\prime \prime}=1.2 \times 10^{16} \mathrm{~cm}$. Also, the proper motion velocity of knot $\mathrm{G}$ is $v_{G}=287 \mathrm{~km} \mathrm{~s}^{-1}$, which is very close to its full spatial velocity because the outflow lies at a very small angle with respect to the plane of the sky.

First, with the $x_{G}, \Delta x_{G}$ and $v_{G}$ values, we use equations (34) and (35) to obtain a period $\tau_{p}=13.3 \mathrm{yr}$ and a shock velocity $v_{s}=44.2 \mathrm{~km} \mathrm{~s}^{-1}$.

Then, taking the knot $\mathrm{G}$ line fluxes from Nisini et al. (2005), applying a reddening correction with their $A_{v}=2.0$ extinction (taking a standard, $E(B-V) / A_{v}=3.1$ extinction curve) and assuming a distance of $400 \mathrm{pc}$ to $\mathrm{HH} 1$, we obtain $L_{H \alpha}=1.77 \times 10^{-4} L_{\odot}$ and $L_{[S I I]}=5.19 \times$ $10^{-4} L_{\odot}$. Using equation (36) with $\Delta v_{0}=v_{s}$, we obtain $\dot{M}_{H \alpha}=7.76 \times 10^{-8} M_{\odot} \mathrm{yr}^{-1}$ and $\dot{M}_{[S I I]}=8.07 \times 10^{-7} M_{\odot} \mathrm{yr}^{-1}$ from the observed $\mathrm{H} \alpha$ and [S II] emission of knot G, respectively. 
These two mass loss rate estimates can be compared with the estimates of Nisini et al. (2005). who (using different methods) find $\dot{M} \approx 6.9 \times 10^{-8} \rightarrow$ $2.4 \times 10^{-7} M_{\odot} \mathrm{yr}^{-1}$ for knot $\mathrm{G}$ of the HH 1 jet. Of our two estimates, we favour the $8.07 \times 10^{-7} M_{\odot} \mathrm{yr}^{-1}$ estimate obtained from the [S II] luminosity. This is because the $[\mathrm{S} \mathrm{II}]$ emission is produced closer to the shock than $\mathrm{H} \alpha$, and the $[\mathrm{S} \mathrm{II}]$ prediction from stationary, 1D shock models is therefore more likely to be applicable to the time-dependent, multidimensional jet flow.

\section{SUMMARY}

We have applied the "center of mass equation of motion" to find the asymptotic behaviour (at large distances from the outflow source) of the internal working surfaces produced by an arbitrary, periodic outflow variability with an ejection velocity $u_{0}(\tau)$ and a density $\rho_{0}(\tau)$. We find the complete asymptotic solution, giving the constant, asymptotic velocity $v_{a}$ and the position-dependent shock velocities and preshock densities of the working surfaces.

We obtain the same position-dependencies that have been found by Raga \& Kofman (1992) using the "ram-presure balance" equation of motion for the working surfaces. However, Raga \& Kofman (1992) were unable to find the relation between the proportionality constants (for the density and shock velocity vs. position) and the ejection variability.

With our full asymptotic solution, we compute the knot properties for two chosen combinations of $u_{0}(\tau)$ and $\rho_{0}(\tau)$ (see $\S 4$ ). We also discuss the "inverse problem" of finding the properties of the ejection from the observational characteristics of the jet knots (see $\S 5$ ). In particular, we derive a very simple expression for estimating the time-averaged mass loss rate of the ejection as a function of the position $x$, the separation $\Delta x$ between successive knots, the spatial velocity $v_{a}$ and the luminosity $L_{\text {line }}$ (in $\mathrm{H} \alpha$ or in the red [S II] lines) of a given knot.

We apply this "inverse problem" to observations of the $\mathrm{HH} 1$ jet (line intensities and extinctions of Nisini et al. 2005 and proper motions of Raga et al. 2017), and find mass loss rates which are similar to the ones of Nisini et al. (2005). This result is nothing short of surprising, given the fact that our mass loss rate determination is completely model-dependent, and comes from a rather eclectic collection of observational characteristics (e.g., including the knot spacing).

This success of obtaining the previously determined mass loss rate is interesting in two different ways:
- it shows in a quite definite way that the interpretation of the chain of knots of the HH 1 jet as internal working surfaces formed by a quasiperiodic outflow variablity is apparently correct,

- it gives us a new method for determing mass loss rates of outflows from young stars, using the spatial velocity, knot spacings and the intensity in a single emission line of the knots along the $\mathrm{HH}$ jet.

Less optimistically, we note that we have determined (through the use of the asymptotic working surface model) the mass loss rate of the $\mathrm{HH} 1$ jet from the $\mathrm{H} \alpha$ and $[\mathrm{S} \quad \mathrm{II}]$ luminosities, obtaining $\dot{M}=7.8 \times 10^{-8}$ and $8.1 \times 10^{-7} M_{\odot} \mathrm{yr}^{-1}$, respectively, which differ by one order of magnitude. This result is in agreement with the results of Nisini et al. (2005) partly because they also obtain a range of mass loss rate determinations which also differ (from each other) by an order of magnitude. This is clearly not a very good situation.

In our "asymptotic working surface model" mass loss rate determinations, the obvious possible reason for the discrepancy between the $\mathrm{H} \alpha$ and [S II] results is the modelling of the emission with steady, planeparallel shock models. As has been already noted in the early literature on modelling $\mathrm{HH}$ objects (see Dopita et al. 1982), the cool tail of the recombination region does not have time to develop fully in $\mathrm{HH}$ shock waves. The resulting "truncation" of the cooling region has a stronger effect on the predicted $\mathrm{H} \alpha$ emission than on the forbidden lines (Raga \& Binette 1991), so that the mass loss rate deduced from the [S II] luminosity (i.e., $\dot{M}=8.1 \times 10^{-7} M_{\odot} \mathrm{yr}^{-1}$ ) is likely to be more reliable.

Also, not only the shocks in working surfaces have non-steady state recombination regions, but also they are not likely to be plane. This is seen in numerical simulations of variable jets (see, e.g., Raga et al. 2007) as well as in high angular resolution observations of $\mathrm{HH}$ jets (see, e.g., Reipurth et al. 2002). It is therefore to be expected that analyses with the assumption of the emission being produced by plane, steady, shocks will not give fully consistent mass loss rate determinations using different emission lines.

We end by noting that there is a lot of indirect evidence that the knot structures along $\mathrm{HH}$ jets are the result of a variable ejection. This evidence is provided by the surprising success of variable jet models at reproducing the observed morphologies, the proper motions and the time-evolution of $\mathrm{HH}$ jets (see, e.g., Castellanos-Ramírez et al. 2018). How- 
ever, convincing observations of a variable ejection from the outflow sources (i,e., in the spectra of the young stars or the protostars ejecting the $\mathrm{HH}$ jets) that can be directly linked to structures along the jets have been elusive. Some observations of the so-called "HH microjets" (with distance scales of $\approx 10^{16} \mathrm{~cm}$ and timescales of $\approx$ a few years) might be showing such a connection (see, e.g., Agra-Amboage et al. 2011). However, for obvious reasons such observations have not been made for the larger scale "normal" $\mathrm{HH}$ jets (with distance scales $\approx 10^{17} \mathrm{~cm}$ and timescales from several decades to $\approx 1000 \mathrm{yr}$ ).

Because of this general lack of direct link to the time-dependence of the outflow source, the details of the ejection variability cannot be determined directly and have to be chosen in a way that results in the production of a jet with the observed characteristics. In particular, while the mean velocity and characteristic period of the variability producing a chain of knots can be satisfactorily constrained by observations of the spatial motion (radial velocities+proper motions) and knot spacing, estimates of the amplitude of the ejection velocity variability depend on less convincing arguments about the excitation of the emission line spectrum of the knots closer to the outflow sources (see $\S 7$ ).

This work was supported by the DGAPA (UNAM) grant IG100218. AC was supported by a DGAPA (UNAM) postdoctoral fellowhip. We thank Pierre Lesaffre (the referee) for helpful comments.

\section{APPENDIX}

\section{A. FITS TO THE LINE EMISSION OF PLANE-PARALLEL SHOCKS}

We approximate the $\mathrm{H} \alpha$ and [S II] $6716+30$ (which we will call "[S II]") line emission of the working surface shocks with the plane-parallel, steady shock models of Hartigan et al. (1987). These lines show the well known scaling:

$$
I_{\text {line }}=n_{\text {pre }} f_{\text {line }}\left(v_{s}\right),
$$

where $I_{\text {line }}$ is the intensity in a given line emerging from the front of the shock, $n_{\text {pre }}$ is the pre-shock ion+atom number density (which in the following we assume is in units of $\left.\mathrm{cm}^{-3}\right)$, and $f_{\text {line }}\left(v_{s}\right)$ is a function of the shock velocity $v_{s}$ which is obtained from the detailed 1D, stationary shock models. For a gas with $90 \% \mathrm{H}$ and $10 \% \mathrm{He}, n_{\text {pre }}=\rho_{\text {pre }} /\left(1.3 m_{H} H\right)$ (with $m_{H}$ being the hydrogen mass), where $\rho_{\text {pre }}$ is the pre-shock density.

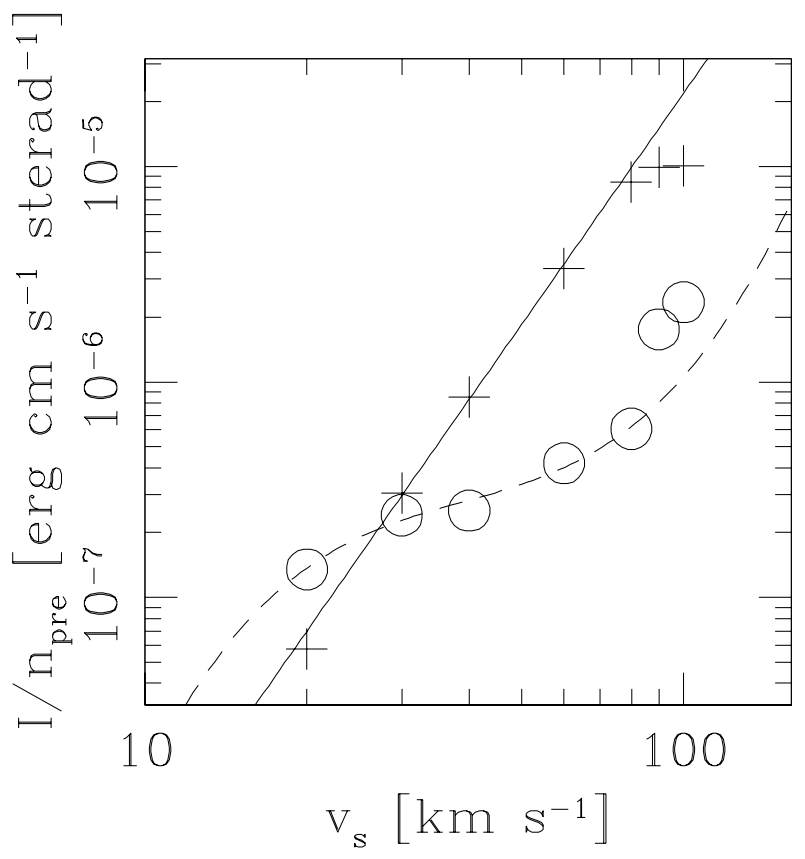

Fig. 2. Predictions of the $\mathrm{H} \alpha$ (crosses) and red [S II] intensities (open circles) as a function of shock velocity $v_{s}$ from the models of Hartigan et al. (1987). The solid and dashed lines show the analytic fits of equations (A38) and (A39), respectively.

In Figure 2, we show the values of $f_{H \alpha}=F_{H \alpha} / n_{\text {pre }}$ and $f_{[S I I]}=F_{[S I I]} / n_{\text {pre }}$ for the $v_{s}=20 \rightarrow 100 \mathrm{~km} \mathrm{~S}^{-1}$ models of Hartigan et al. (1987). For the shocks in the $v_{s}=20 \rightarrow 80 \mathrm{~km} \mathrm{~s}^{-1}$ range, the $\mathrm{H} \alpha$ flux closely follows the power law:

$$
\log _{10} f_{H \alpha}=3.57 \log _{10} v_{s}-11.84,
$$

with $v_{s}$ in $\mathrm{km} \mathrm{s}^{-1}$ and $f_{H \alpha}$ in erg $\mathrm{cm} \mathrm{s}^{-1}$.

The red [S II] emission has a more complicated dependence with $v_{s}$, and in order to fit it with power laws one has to specify limited shock velocity ranges. We fit a cubic polynomial to the "log-log" relation in the $v_{s}=20 \rightarrow 80 \mathrm{~km} \mathrm{~s}^{-1}$ range, obtaining:

$$
\begin{aligned}
\log _{10} f_{[S I I]}= & 4.28\left(\log _{10} v_{s}-1.59\right)^{3}+ \\
& 0.70 \log _{10} v_{s}-7.67,
\end{aligned}
$$

with $v_{s}$ in $\mathrm{km} \mathrm{s}^{-1}$ and $f_{[S I I]}$ in erg $\mathrm{cm} \mathrm{s}^{-1}$. This relation provides a smooth interpolation between the predictions of the $20 \rightarrow 80 \mathrm{~km} \mathrm{~s}^{-1}$ shock models (see Figure 2).

\section{REFERENCES}

Agra-Amboage, V., Dougados, C., Cabrit, S., \& Reunanen, J. 2011, A\&A, 532, A59 
Cantó, J., Raga, A. C., \& D'Alessio, P. 2000, MNRAS, 313,656

Cantó, J. \& Raga, A. C. 2003, RMxAA, 39, 261

Castellanos-Ramírez, A., Raga, A. C., \& RodríguezGonzález, A. 2018, ApJ, 867, 29

Dopita, M. A., Binette, L., \& Schwartz, R. D. 1982, ApJ, 261,183

Hansen, E. C., Frank, A., Hartigan, P., \& Lebedev, S. V. 2017, ApJ, 837, 143

Hartigan, P., Raymond, J., \& Hartmann, L. 1987, ApJ, 316,323

Kofman, L. \& Raga, A. C. 1992, ApJ, 390, 359

Nisini, B., Bacciotti, F., Giannini, T., et al. 2005, A\&A, 441, 159

Podio, L., Bacciotti, F., Nisini, B., et al. 2006, A\&A, 456, 189
Raga, A. C., Cantó, J., Binette, L., \& Calvet, N. 1990, ApJ, 364, 601

Raga, A. C. \& Binette, L. 1991, RMxAA, 22, 265

Raga, A. C. \& Kofman, L. 1992, ApJ, 386, 222

Raga, A. C. \& Cantó, J. 1998, RMxAA, 34, 73

Raga, A. C., de Colle, F., Kajdiĉ, P., Esquivel, A., \& Cantó, J. 2007, A\&A, 465, 879

Raga, A. C., Reipurth, B., Esquivel, A., et al. 2017, RMxAA, 53, 485

Rees, M. J. 1978, MNRAS, 184, 61

Reipurth, B., Heathcote, S., Morse, J., Hartigan, P., \& Bally, J. 2002, AJ, 123, 362

Roberts, D. A. 1986, ApJ, 300, 568

Teşileanu, O., Matsakos, T., Massaglia, S., et al. 2014, A\&A, 562, 117

Wilson, M. J. 1984, MNRAS, 209, 923

J. Cantó and A. Castellanos-Ramírez: Instituto de Astronomía, Ap. 70-468, 04510 Cd. Mx., México.

A. C. Raga: Instituto de Ciencias Nucleares, Universidad Nacional Autónoma de México, Ap. 70-543, 04510 CDMX, México, (raga@nucleares.unam.mx).

A. C. Raga: Instituto de Investigación en Ciencias Físicas y Matemáticas, USAC, Ciudad Universitaria, Zona 12, Guatemala, (raga@nucleares.unam.mx). 